\title{
Karlsruhe Brainstormers 2000 Team Description
}

\author{
Martin Riedmiller, Artur Merke, David Meier, Andreas Hoffmann, Alex Sinner, \\ Ortwin Thate
}

Institut für Logik, Komplexität und Deduktionssysteme University of Karlsruhe, D-76128 Karlsruhe, FRG

\section{Introduction}

The main motivation behind the Karlsruhe Brainstormer's effort in the robotic soccer simulation league of the RoboCup project is to develop and to apply Reinforcement Learning (RL) techniques in complex domains. Our long term goal is to have a learning system which is able to learn by itself the best winning behaviour. The soccer simulation domain allows more than $(108 \times 50)^{23}$ different positionings of the 22 players and the ball - the complete state space considering object velocities and player's stamina is magnitudes larger. In every cycle, an agent can choose between more than 300 basic commands (parametrized turns and dashes), which makes a choice of $300^{11}$ joint actions for the team per cycle! A problem of such complexity is a big challenge for today's RL methods; in the Brainstormers project we are investigating methods to practically handle learning problems of such size.

\section{Special Team Features}

The outstanding features of the Karlsruhe Brainstormers Agent are its approach to learn the moves with RL methods and the search algorithms used on the strategy level for planning complex coordinated team moves such as pass chains. It is our aim to have one day an agent which has learned its whole behaviour through RL methods. For further information see [1], [2], [3].

\section{World Model}

Construction of an own world model was not a main focus of our development. Therefore we decided to use parts of the world model sources of the CMU team from 1999 [4]. This world model is hidden behind our own interface, so we can switch oine day to our own world model. We also added some communication, so that players can exchange their knowledge about positions of other players (which considerably improves the quality of the world model information). From the tactics point of view it is also interesting to know how the players play with full and noiseless world model information. To this end we use a sligthly modified soccer server which communicates the complete noiseless state information to our players. As the construction of the world model is hidden behind an interface we

P. Stone, T. Balch, and G. Kraetzschmar (Eds.): RoboCup 2000, LNAI 2019, pp. 485-488, 2001.

(C) Springer-Verlag Berlin Heidelberg 2001 
can choose to play with or without a complete world model. For the development of RL moves we also use another patch of the official server which allows us to run the server faster i.e. without waiting the $100 \mathrm{~ms}$ each cycle, but just waiting until the agents have sent their commands. In conjunction with the full information patch we have a powerful learn development tool at our disposal.

\section{Coach}

The Brainstormers 2000 client did not make use of an online coach, altough a coach is used to automate unsupervised playing of games. This allows us to test our team in say ten or more games against several other teams and gather statistics.

Another tool which uses coach commands is Framview2d. This is a viewer for moved two dimensional graphics. It uses frames for the representation of geometrical data (like points, lines, circles). For example, it is possible to put contours of a robot into a frame and later on just to specify the frame position and angle, all coordinates conversion are done automaticly. For a longer list of features see http://i11www.ira.uka.de/ ^amerke/frameview. By now there is a module which can be used instead of the official soccer monitor of the simulation league. By using the zoom facility it can be used e.g. for monitoring or debugging of moves. So it is possible to watch a kick sequence produced by our RL kick move or the turns and dashes during our RL intercept ball move. We also use it to edit game situations which can be saved and then reproduced with our coach tool. It can be easily extended to visualize other $2 \mathrm{~d}$ worlds, e.g. it would be a straightforward task to use it as a monitor of a middle/small size league robot.

\section{Communication}

Our agents communicate basically two different kinds of information : first, if an agent makes an important decision affecting teamplay (such as a pass), he communicates this decision so that the receiving player knows he will receive a pass and can therefore act faster than his opponents. Otherwise the agents simply communicate their world information so every player is able to have a better view of the world.

\section{Skills}

Most of the nontrivial skills are learned with RL methods. We refer to them as moves. A move is a sequence of basic actions. Since each move has a clearly defined goal, it is straightforward to apply RL methods to solve the problem of getting an optimal decision policy. We applied Real-Time Dynamic Programming methods that solve the learning problem by incrementally approximating an optimal value function by repeated control trials. Since the state space is continuous, a feedforward neural network is used to approximate the value function.[1] 


\section{Strategy}

Altough it is our long-term goal to learn a high level strategy with RL methods, our research is currently not advanced enough to use a learned strategy efficiently in our client. Nevertheless this does not mean we did not develop any strategy. Basically the client chooses from two different strategies : a strategy which considers that he has the ball and a strategy for positioning if he doesn't have the ball. In the latter case the team switches the formation according to whether it is attacking, defending or in midfield play. In this formation every player is assigned a radius of action in which he may move around to perform passive tasks such as defend against an attacking opponent, receive a pass etc. If the player is in possession of the ball, he uses a planning algorithm which searches for pass chains. If no good passes can be played (or no goal shot), he looks for alternatives like dribbling, holding the ball etc.

\section{Team Development}

The development team for the Karlsruhe Brainstormers 2000 client consisted of 6 people. We meet once a week to discuss more or less important matters and coordinate the client development. After the championships in Stockholm 1999 we decided to entirely redesign our client, so we rewrote most of the code from scratch, implementing advanced software engineering concepts such as object handlers. The client is thoroughly modularized, which makes it easy to test and implement new strategies, moves etc. The decision process is organised hierarchically : on the top level a strategy is selected at the beginnig of the game (e.g. goalie or 'normal' player). This strategy selects a policy according to the playmode and overall situation. The policy defines how the client has to behave, i.e. he selects a move. A move is a sequence of basic actions, so it returns an action which is sent to the server. Furthermore, since four types of commands can be sent in parallel to the server (main commands, turn-neck commands, say commands and change-view commands) we have four parallel decision chains.

To coordinate our development we use CVS, a powerful revision control system. To generate documentation from our code we use doxygen. Everything is developed on Linux machines.

Team Leader: Dr. Martin Riedmiller Team Members:

Artur Merke

- Computer Sciences

- Germany

- graduate student

- did attend the competition

David Meier

- Computer Sciences

- Germany 
- graduate student

- did attend the competition

Andreas Hoffmann

- Computer Sciences

- Germany

- undergraduate student

- did attend the competition

Alex Sinner

- Computer Sciences

- Luxembourg

- undergraduate student

- did attend the competition

Ortwin Thate

- Computer Sciences

- Germany

- undergraduate student

- did attend the competition

Web page http://www.karlsruhe-brainstormers.de

\section{Conclusion}

Our success (vice-champion) at the championships im Melbourne showed us that the RL approach is very promising. We plan to extend its use to larger scale problems such as positioning, planning and teamplay. Distributed RL will be one of our main research interests for the Brainstormers 2001 project. And yes, we will be participating at the RoboCup world championships in Seattle 2001 !

Finally we want to thank all our friends, family and all the people who supported us in any way.

See you in Seattle!

\section{References}

1. M. Riedmiller, A. Merke, D. Meier, A. Hoffmann, A. Sinner, C. Kill and R. Ehrmann. Karlsruhe Brainstormers - A Reinforcement Learning approach to robotic soccer. In A. Jennings, P. Stone, editors, RoboCup-2000: Robot Soccer World Cup $I V$, LNCS Springer Verlag, Berlin, 2000.

2. A. G. Barto, S. J. Bradtke, and S. P. Singh. Learning to act using real-time dynamic programming. Artificial Intelligence, (72):81-138, 1995.

3. M. Riedmiller. Concepts and facilities of a neural reinforcement learning control architecture for technical process control. Journal of Neural Computing and Application, 8:323-338, 2000.

4. Peter Stone, Patrick Riley and Manuela Veloso. The CMUnited-99 Champion Simulator Team In M. Veloso, E. Pagello and H. Kitano, editors, RoboCup-99: Robot Soccer World Cup III. Springer Verlag, Berlin, 1999. 